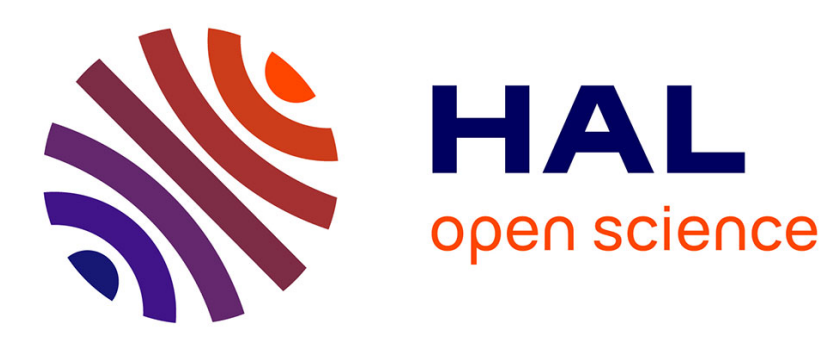

\title{
Voltage Sags Estimation in Three-Phase Systems using Unconditional Maximum Likelihood Estimation
}

\author{
Vincent V. Choqueuse, A Belouchrani, Guillaume Bouleux, Mohamed \\ Benbouzid
}

\section{- To cite this version:}

Vincent V. Choqueuse, A Belouchrani, Guillaume Bouleux, Mohamed Benbouzid. Voltage Sags Estimation in Three-Phase Systems using Unconditional Maximum Likelihood Estimation. 40th IEEE International Conference on Acoustics, Speech and Signal Processing (ICASSP 2015), IEEE, Apr 2015, Brisbane, Australia. pp.2869 - 2873, 10.1109/ICASSP.2015.7178495 . hal-01146218

\section{HAL Id: hal-01146218 https://hal.science/hal-01146218}

Submitted on 28 Aug 2015

HAL is a multi-disciplinary open access archive for the deposit and dissemination of scientific research documents, whether they are published or not. The documents may come from teaching and research institutions in France or abroad, or from public or private research centers.
L'archive ouverte pluridisciplinaire HAL, est destinée au dépôt et à la diffusion de documents scientifiques de niveau recherche, publiés ou non, émanant des établissements d'enseignement et de recherche français ou étrangers, des laboratoires publics ou privés. 


\title{
VOLTAGE SAGS ESTIMATION IN THREE-PHASE SYSTEMS USING UNCONDITIONAL MAXIMUM LIKELIHOOD ESTIMATION
}

\author{
V. Choqueuse ${ }^{(1)}$, A. Belouchrani ${ }^{(2)}$, G. Bouleux ${ }^{(3)}$ and M.E.H. Benbouzid $^{(1)}$ \\ (1) University of Brest, LBMS, EA4325, Brest Cedex 03, CS 93837, France \\ ${ }^{(2)}$ Ecole Nationale Polytechnique d'Alger, Algier, 16200, Algeria \\ (3) Université de Lyon, Université de Saint Etienne, DISP, EA 4570, Insa de Lyon, France
}

\begin{abstract}
This paper focuses on the estimation of voltage sags in threephase power systems. Specifically, it proposes a new approach for estimating the amplitude and phase angle of the sag based on the Unconditional Maximum Likelihood technique. As opposed to other techniques, this approach is well suited for signals with amplitude and/or phase modulation such as those encountered in smart grid applications. Simulation and experimental results illustrate the effectiveness of the proposed approach.
\end{abstract}

Index Terms- Maximum Likelihood Estimation, Power systems, Smart Grid Monitoring, Voltage sags.

\section{INTRODUCTION}

Analysis of electrical signals plays a key role in many engineering applications. Application includes, for example, electrical machines monitoring based on current signature analysis [1] or smart-grid monitoring [2,3]. From a signal processing viewpoint, one particularity of the electrical signal is its multidimensional nature. Indeed, electrical signals are usually composed of three components called "phases". In ideal conditions, each phase can be modelled by a simple sine wave with constant amplitude and frequency $\left(f_{0}=60 \mathrm{~Hz}\right.$ in the US) and $2 \pi / 3$ phase-shift between consecutive phases. In practical systems, many deviations can occur such as amplitude/frequency variations and three-phase unbalance [4]. In particular, three-phase unbalance can be composed of amplitude drops and phase angle-jumps. These phenomenona, which are called voltage sags, can be damaging for electrical machines or grid equipments. For this reason, the monitoring of voltage sags is of main concern in modern power systems.

Several techniques have been proposed in the literature for estimating the amplitude and phase angle parameters. The IEEE 1564 standard recommends the use of the RMS value [5]. Another simple and commonly used technique is based on the Peak Voltage. Nevertheless, the Root Mean Square (RMS) and Peak Voltage techniques assume noiseless signals and do not provide any estimate of the phase angle parameter. To overcome these limitations, several authors propose to estimate the amplitude and phase angle from the Discrete Fourier Transform (DFT) component at frequency $f_{0}[3,4]$. The main drawback is that this technique assumes a constant and perfectly known frequency $f_{0}$. For signals with frequency offset, improvement can be obtained by using extensions of the periodogram [6-11] but these techniques do not perform well under non-stationary conditions. Under non-stationary conditions, techniques based on adaptive filtering have been proposed in $[12,13]$. However, the aforementioned techniques are suboptimal since they treat each phase independently. In [14], a Maximum Likelihood (ML) technique has been proposed for estimating the amplitude parameters from the three-phase signal, but it is not suited for systems with phase angle jumps. In [15], the system parameters are estimated from the symmetrical components. However, this technique assumes constant parameters and is not suited for signals with amplitude/frequency variations.

In this paper, a new technique for estimating the sag amplitude and phase angle parameters is proposed. This technique corresponds to the Unconditional Maximum Likelihood estimator for the Rayleigh fading sinusoid model. This paper is organized as follows. Section 2 presents the three-phase signal model, Section 3 describes the proposed estimator, and Section 4 focuses on the simulation results.

\section{THREE-PHASE SIGNAL MODEL}

In three-phase systems, voltages (or currents) can be described by the following equation (see [16])

$$
x_{k}[n]=\sqrt{2} d_{k} a[n] \cos \left(\phi[n]+\varphi_{k}\right)+b_{k}[n]
$$

where $x_{k}[n]$ and $b_{k}[n]$ correspond to the voltage and noise in the $k^{\text {th }}$ phase $(k=0,1,2)$. The components $a[n]$ and $\phi[n]$ correspond to the instantaneous scaling factor for the amplitude and instantaneous phase offset, respectively. As compared to other studies, these components are not assumed to be constant or linear. The parameters $d_{k} \geq 0$ and $\varphi_{k}$ correspond to the amplitude and phase angle, respectively. Note that under nominal conditions, $\varphi_{k}=2 k \pi / 3$ [16]. Using ma- 
trix notations, (1) can expressed as

$$
\mathbf{x}[n]=\mathbf{H}(\Theta) \boldsymbol{\alpha}[n]+\mathbf{b}[n]
$$

where:

- $\Theta=\left\{d_{0}, d_{1}, d_{2}, \varphi_{0}, \varphi_{1}, \varphi_{2}\right\}$ contains the amplitude and phase angle parameters,

- $\mathbf{x}[n]$ and $\mathbf{b}[n]$ are $3 \times 1$ column vectors defined as

$$
\mathbf{x}[n]=\left[\begin{array}{c}
x_{0}[n] \\
x_{1}[n] \\
x_{2}[n]
\end{array}\right] \quad \mathbf{b}[n]=\left[\begin{array}{c}
b_{0}[n] \\
b_{1}[n] \\
b_{2}[n]
\end{array}\right],
$$

- $\mathbf{H}(\Theta)$ is a $3 \times 2$ matrix which is defined as

$$
\mathbf{H}(\Theta)=\left[\begin{array}{ll}
d_{0} \cos \left(\varphi_{0}\right) & -d_{0} \sin \left(\varphi_{0}\right) \\
d_{1} \cos \left(\varphi_{1}\right) & -d_{1} \sin \left(\varphi_{1}\right) \\
d_{2} \cos \left(\varphi_{2}\right) & -d_{2} \sin \left(\varphi_{2}\right)
\end{array}\right]
$$

- $\boldsymbol{\alpha}[n]$ is a $2 \times 1$ vector containing the direct and quadrature components, i.e.

$$
\boldsymbol{\alpha}[n] \triangleq \sqrt{2}\left[\begin{array}{c}
a[n] \cos (\phi[n]) \\
a[n] \sin (\phi[n])
\end{array}\right]
$$

The goal of this paper is to estimate $\Theta$ from $N$ measurements without knowing $\boldsymbol{\alpha}[n]$. In order to estimate these parameters, we make use of the following assumptions:

- AS1) The additive noise is a zero-mean, white Gaussian noise with covariance matrix $E\left[\mathbf{b}[n] \mathbf{b}^{T}[n]\right]=\sigma^{2} \mathbf{I}$ i.e. $\mathbf{b}[n] \sim \mathcal{N}\left(0, \sigma^{2} \mathbf{I}\right)$.

- AS2) The instantaneous amplitude and phase are independent of each other. Furthermore, $a[n]$ is Rayleigh distributed and $\phi[n]$ is uniformly distributed in $[0,2 \pi$. Under these assumptions, $\boldsymbol{\alpha}[n] \sim \mathcal{N}\left(0, \sigma_{\alpha}^{2} \mathbf{I}\right)[17, \mathrm{Ex}-$ ample 5.5]. Finally, without loss of generality, we assume that the variance of the $k^{t h}$ phase is only driven by the amplitude $d_{k}$ i.e. $\sigma_{\alpha}^{2}=1$.

\section{AMPLITUDE AND ANGLE ESTIMATION.}

In this section, we propose a two-step approach for estimating $\Theta$. First, we estimate the mixing matrix $\mathbf{H}$ from $\mathbf{X}$ using the Unconditional Maximum Likelihood estimator, then we resort to the invariance principle of the Maximum Likelihood to estimate $\Theta$ from $\widehat{\mathbf{H}}$.

\subsection{Estimation of $\mathbf{H}$}

\subsubsection{Maximum Likelihood Estimation}

Under AS1-AS2), it can be checked that $\mathbf{x}[n] \sim \mathcal{N}(0, \mathbf{R})$ where $\mathbf{R}=\mathbf{H H}^{T}+\sigma^{2} \mathbf{I}$. Therefore, the ML Estimates of $\mathbf{H}$ and $\sigma^{2}$ can be obtained by minimizing

$$
\mathcal{L}\left(\mathbf{X} ; \mathbf{H}, \sigma^{2}\right)=\log (\operatorname{det}(\mathbf{R}))+\operatorname{Tr}\left[\mathbf{R}^{-1} \widehat{\mathbf{R}}\right]
$$

where $\mathbf{X} \triangleq \mathbf{x}[0], \cdots, \mathbf{x}[N-1]$ and $\widehat{\mathbf{R}}$ is the data covariance matrix which is defined as

$$
\widehat{\mathbf{R}} \triangleq \frac{1}{N} \sum_{n=0}^{N-1} \mathbf{x}[n] \mathbf{x}^{T}[n]
$$

Moreover, it is known that the minimum is reached at [18]

$$
\begin{aligned}
\widehat{\mathbf{H}} & =\widehat{\mathbf{U}} \widehat{\boldsymbol{\Lambda}}^{\frac{1}{2}} \mathbf{V} \\
\widehat{\sigma}^{2} & =\lambda_{0}
\end{aligned}
$$

where

- $\widehat{\Lambda}$ is a $2 \times 2$ diagonal matrix with diagonal elements $\lambda_{2}-\lambda_{0}$ and $\lambda_{1}-\lambda_{0}$, where $\lambda_{2} \geq \lambda_{1} \geq \lambda_{0}$ are the eigenvalues of $\widehat{\mathbf{R}}$,

- $\widehat{\mathbf{U}}$ is a $3 \times 2$ matrix containing the eigenvectors associated with the two largest eigenvalues of $\widehat{\mathbf{R}}$,

- $\mathbf{V}$ is an arbitrary $2 \times 2$ orthogonal matrix.

In the following paragraph, the orthogonal matrix $\mathbf{V}$ is determined by adding constraints to the structure of $\widehat{\mathbf{H}}$.

\subsubsection{Determining the Orthogonal Matrix $\boldsymbol{V}$}

Let us define the $3 \times 2$ matrix $\mathbf{W}$ as

$$
\mathbf{W} \triangleq \widehat{\mathbf{U}} \widehat{\Lambda}^{\frac{1}{2}}=\left[\begin{array}{ll}
w_{00} & w_{01} \\
w_{10} & w_{11} \\
w_{20} & w_{21}
\end{array}\right]
$$

As $\mathbf{V}$ is a $2 \times 2$ orthogonal matrix, $\mathbf{V} \in\left\{\mathbf{V}_{0}(\theta), \mathbf{V}_{1}(\theta)\right\}$ where

$$
\begin{aligned}
& \mathbf{V}_{0}(\theta) \triangleq\left[\begin{array}{cc}
\cos (\theta) & -\sin (\theta) \\
\sin (\theta) & \cos (\theta)
\end{array}\right] \\
& \mathbf{V}_{1}(\theta) \triangleq\left[\begin{array}{cc}
\cos (\theta) & \sin (\theta) \\
\sin (\theta) & -\cos (\theta)
\end{array}\right]
\end{aligned}
$$

To determine $\mathbf{V}$, we impose two natural constraints on $\Theta$ (see Fig.1).

- $\mathcal{C}_{1}$ : The first phase corresponds to the reference phase i.e. $\varphi_{0}=0$.

- $\mathcal{C}_{2}$ : The second phase belongs to $[0 \pi]$ i.e. $0 \leq \varphi_{1} \leq \pi$.

The second constraint, $\mathcal{C}_{2}$, is motivated by the fact that under nominal conditions $\varphi_{1}=2 \pi / 3$. Deviations from this value are usually smaller than $\pi / 3$ [19]. Using $\mathcal{C}_{1}$ and $\mathcal{C}_{2}$ in (8), it can be shown that $\mathbf{H}(\Theta)$ has the following structure

$$
\mathbf{H}(\Theta)=\left[\begin{array}{cc}
\times & 0 \\
\times & \leq 0 \\
\times & \times
\end{array}\right]
$$




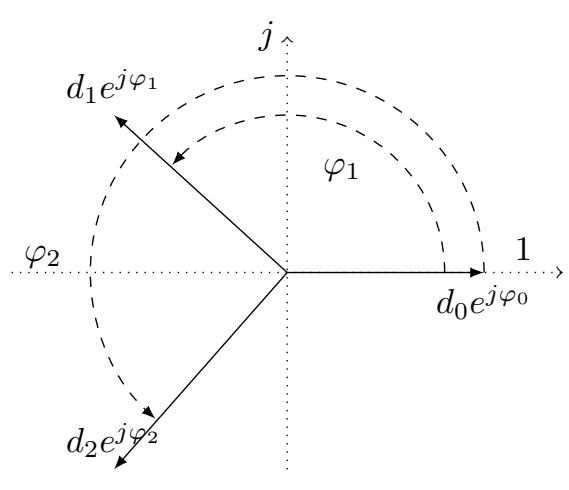

Fig. 1: Representation of $d_{k} e^{j \varphi_{k}}$ in the complex plane. The reference phase $\varphi_{0}$ is set to 0 (constraint $\mathcal{C}_{1}$ ) and $\varphi_{1} \in[0, \pi]$ (constraint $\mathcal{C}_{2}$ ).

By imposing the constraint $\mathcal{C}_{1}$ on $\mathbf{W V}_{0}(\theta)$, we obtain $\widehat{\theta}=$ $\arctan (\beta)+k \pi$, where $k=1$ if $w_{00}<0$ and $k=0$ elsewhere, and

$$
\beta \triangleq \frac{w_{01}}{w_{00}}
$$

It can be verified that $\mathcal{C}_{1}$ leads to the same estimate when applied on $\mathbf{W} \mathbf{V}_{1}(\theta)$. Using trigonometric identities [20, Part 1.624 , identities (7) and (8)], $\mathbf{V}_{0}(\widehat{\theta})$ and $\mathbf{V}_{1}(\widehat{\theta})$ can be simplified as

$$
\begin{aligned}
& \mathbf{V}_{0}(\widehat{\theta})=\frac{\operatorname{sgn}\left(w_{00}\right)}{\sqrt{1+\beta^{2}}}\left[\begin{array}{cc}
1 & -\beta \\
\beta & 1
\end{array}\right] \\
& \mathbf{V}_{1}(\widehat{\theta})=\frac{\operatorname{sgn}\left(w_{00}\right)}{\sqrt{1+\beta^{2}}}\left[\begin{array}{cc}
1 & \beta \\
\beta & -1
\end{array}\right]
\end{aligned}
$$

where $\operatorname{sgn}($.$) is the sign function. The matrix indeterminacy$ can be removed by using $\mathcal{C}_{2}$. Indeed, we obtain

$$
\widehat{\mathbf{V}}= \begin{cases}\mathbf{V}_{0}(\widehat{\theta}) & \text { if } w_{11} \operatorname{sgn}\left(w_{00}\right) \leq w_{10} \operatorname{sgn}\left(w_{00}\right) \beta \\ \mathbf{V}_{1}(\widehat{\theta}) & \text { elsewhere }\end{cases}
$$

\subsection{Estimation of $\Theta$}

In this subsection, we resort to the invariance principle of the Maximum Likelihood to estimate $\Theta$. First, we derive an expression to compute $\Theta$ from $\mathbf{H}$, then we use this expression to estimate $\Theta$ from $\widehat{\mathbf{H}}$.

Let us define $\mathbf{e}_{k}$ the unit column vector which is " 1 " in the $k^{t h}$ line and zero elsewhere. Using the definitions of $\mathbf{H}(\Theta)$, it can be shown that

$$
\mathbf{e}_{k}^{T} \mathbf{H}(\Theta)=\left[d_{k} \cos \left(\varphi_{k}\right)-d_{k} \sin \left(\varphi_{k}\right)\right] .
$$

Then, let us define the complex scalar $z_{k}$ as

$$
z_{k} \triangleq \mathbf{e}_{k}^{T} \mathbf{H}(\Theta)\left[\begin{array}{c}
1 \\
-j
\end{array}\right]
$$

where $j$ is the imaginary unit. Using (15) in (16), it can be readily checked that $z_{k}=d_{k} e^{j \varphi_{k}}$. Therefore, the value of the amplitude and phase angle parameters can be computed as

$$
\begin{aligned}
d_{k} & =\left|z_{k}\right| \\
\varphi_{k} & =\arg \left[z_{k}\right]
\end{aligned}
$$

where $|$.$| and \arg [$.$] correspond to the complex modulus and$ argument, respectively. In practise $z_{k}$ is unknown and must be replaced by its estimate in (17), where the estimate of $z_{k}$ is given by

$$
\widehat{z}_{k}=\mathbf{e}_{k}^{T} \widehat{\mathbf{U}} \widehat{\Lambda}^{\frac{1}{2}} \widehat{\mathbf{V}}\left[\begin{array}{c}
1 \\
-j
\end{array}\right]
$$

\section{SIMULATION AND EXPERIMENTAL RESULTS}

\subsection{Simulation Results}

Herein, the performances of the proposed approach are compared with respect to those of the RMS technique. For each technique, the Mean Square Error (MSE) is evaluated through 10000 Monte Carlo simulations. The unknown parameters are arbitrary set to $\Theta=\left\{1,0.5,1.8,0^{\circ}, 131.5^{\circ}, 234.2^{\circ}\right\}$. The statistical performances are analysed for different data lengths and signal-to-noise ratios (SNRs), where $\mathrm{SNR}=$ $10 \log _{10}\left(\sum_{k=0}^{2} d_{k}^{2} /\left(3 \sigma^{2}\right)\right)$. The Cramer Rao bounds are also computed. These bounds are derived from the inverse of the Fisher information Matrix, $\mathbf{I}(\Omega)$, where the $(u, v)^{t h}$ element of $\mathbf{I}(\Omega)$ is given by [21]

$$
[\mathbf{I}(\Omega)]_{u v}=\frac{N}{2} \operatorname{Tr}\left[\mathbf{R}^{-1}(\Omega) \frac{\partial \mathbf{R}(\Omega)}{\partial \Omega_{u}} \mathbf{R}^{-1}(\Omega) \frac{\partial \mathbf{R}(\Omega)}{\partial \Omega_{v}}\right],
$$

the set $\Omega=\left\{d_{0}, d_{1}, d_{2}, \varphi_{1}, \varphi_{2}, \sigma^{2}\right\}$ contains the unknown parameters, and $\Omega_{u}$ corresponds to the $u^{t h}$ element of $\Omega$. Note that $\varphi_{0}$ is excluded from $\Omega$ since it is assumed to be known.

In the following paragraphs, we only focus on the estimation of the amplitude parameters, $d_{k}$, since the RMS technique does not provide any estimate of the phase-angle parameters. Figure 2a shows the MSE and CRB versus SNR for $N=100$ samples. We observe that the MSE of the proposed technique is close to the CRB even though it is known that the Unconditional Maximum Likelihood is not efficient at finite number of samples [22]. Furthermore, we note that the proposed technique outperforms the RMS estimator for low and moderate SNR. These two estimators seem to perform equally well as $N$ increased. Theoretically, one can show that these two estimators have similar expressions when $\mathrm{SNR} \rightarrow \infty$. Figure $2 \mathrm{~b}$ presents the MSE and CRB versus $\mathrm{N}$ when $\mathrm{SNR}=20 \mathrm{~dB}$. We observe that the MSE and CRB are really close in the asymptotic region, which corroborates the fact that the Unconditional Maximum Likelihood reaches the CRB when $N \rightarrow \infty$. 


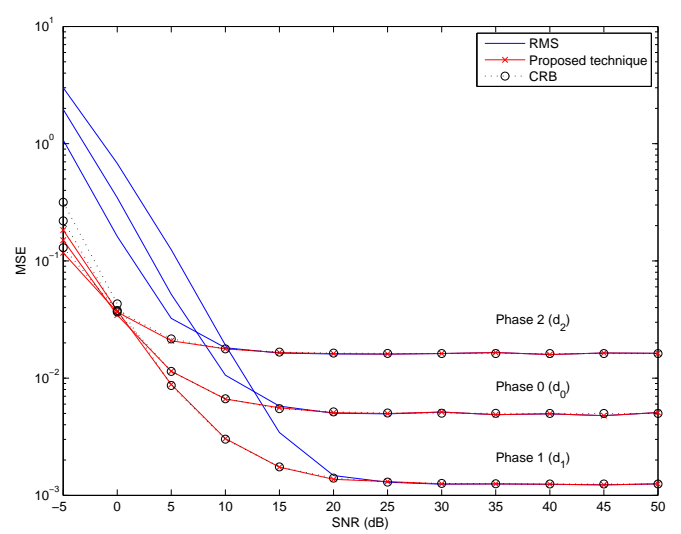

(a) Mean Square Error versus SNR $(N=100)$.

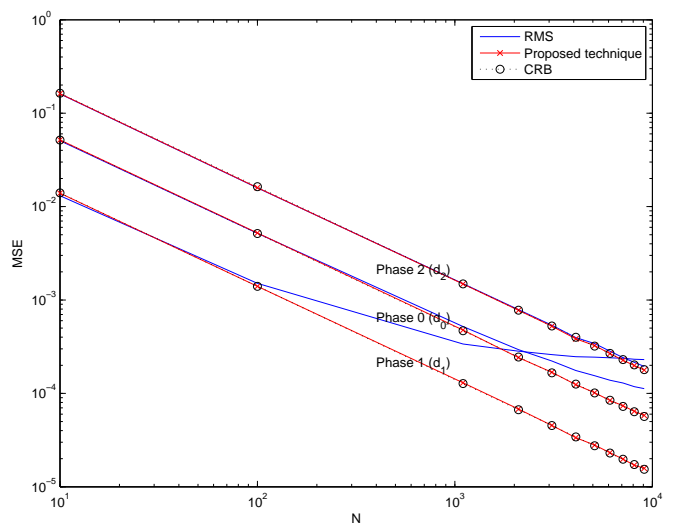

(b) Mean Square Error versus N $(\mathrm{SNR}=20 \mathrm{~dB})$.

Fig. 2: Comparison of the proposed technique and RMS one for amplitude estimation.

\subsection{Experimental Results}

The proposed algorithm is applied to experimental threephase signals under dynamic conditions. Experimental signals come from the DOE/EPRI National Database of Power System Events. The sampling rate is equal to $F_{e}=960 \mathrm{~Hz}$ and the nominal frequency is $f_{0}=60 \mathrm{~Hz}$. To track the variations of the amplitude and phase angle parameters, the data covariance matrix in (7) is replaced by the sliding covariance matrix $\mathbf{R}[n]$, which is defined as

$$
\mathbf{R}[n] \triangleq \frac{1}{L} \sum_{l=0}^{L-1} \mathbf{x}[n-l] \mathbf{x}^{T}[n-l]
$$

The window length is set to $L=32$ samples. Regarding the computational complexity, the most difficult task relies on the eigenvalues decomposition of each covariance matrix $\mathbf{R}[n]$. However, it should be mentioned that this decomposition can be obtained in closed form as exposed in [14]. Figure 3 a shows the voltage signal corresponding to the event 855 (lightning). To deeply characterize this voltage sag, Figures $3 \mathrm{~b}$ and $3 \mathrm{c}$ present the amplitude and phase angle param-

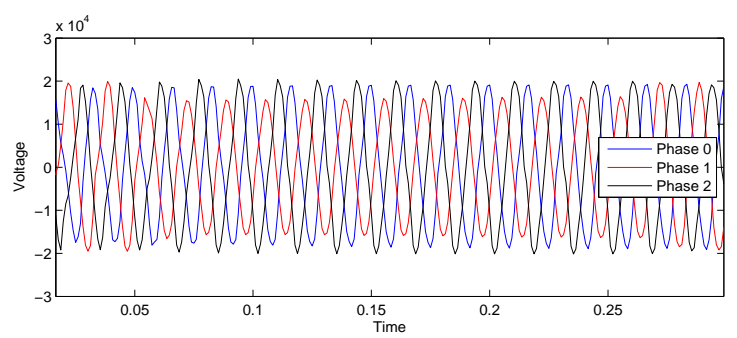

(a) Voltage signal

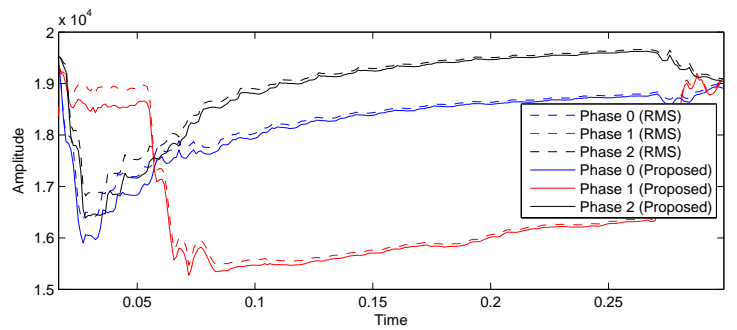

(b) Estimated amplitude parameters.

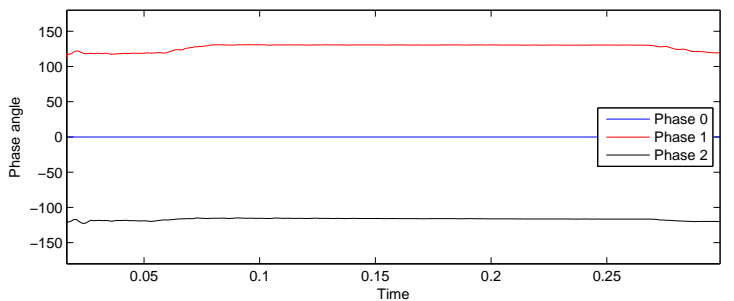

(c) Estimated phase angle parameters.

Fig. 3: Amplitude and phase angle estimation with experimental signals.

eters obtained with the proposed technique. The RMS amplitude estimates are also shown for comparison. We see that the voltage sag both modifies the amplitude and the phase angle parameters $\left(\approx-10^{\circ}\right)$. Furthermore, we observe that the RMS amplitudes are slightly higher than those obtained with the proposed technique.

\section{CONCLUSION}

This paper considers the problem of voltage sags estimation in three-phase systems. It describes a new algorithm based on the Unconditional Maximum Likelihood for estimating the amplitude and phase angle parameters. This algorithm first performs an eigenvalue decomposition of the data covariance matrix, and then apply an orthogonal transformation. Simulations show that the proposed technique performs well under noisy conditions even for a small number of samples. Simulations also show that the proposed technique outperforms the RMS amplitude estimator recommended by the IEEE 1564 Standard. Finally, experimental simulation demonstrates that the proposed estimator can be easily adapted to track the variations of the amplitude and phase angle parameters. 


\section{REFERENCES}

[1] M. Benbouzid, "A review of induction motors signature analysis as a medium for faults detection," IEEE Transactions on Industrial Electronics, vol. 47, no. 5, pp. 984-993, Oct 2000.

[2] P. Top, M. Bell, E. Coyle, and O. Wasynczuk, "Observing the power grid: Working toward a more intelligent, efficient, and reliable smart grid with increasing user visibility," Signal Processing Magazine, IEEE, vol. 29, no. 5, pp. 24-32, Sept 2012.

[3] G. Giannakis, V. Kekatos, N. Gatsis, S.-J. Kim, H. Zhu, and B. Wollenberg, "Monitoring and optimization for power grids: A signal processing perspective," Signal Processing Magazine, IEEE, vol. 30, no. 5, pp. 107128, Sept 2013.

[4] M. H. Bollen, Understanding power quality problems. IEEE press New York, 2000.

[5] "IEEE Guide for Voltage Sag Indices," IEEE Std 15642014, pp. 1-59, June 2014.

[6] M. Wang and Y. Sun, "A practical, precise method for frequency tracking and phasor estimation," IEEE Transactions on Power Delivery, vol. 19, no. 4, pp. 15471552, Oct 2004.

[7] A. G. Phadke and J. S. Thorp, Synchronized phasor measurements and their applications. Springer, 2008.

[8] P. Castello, M. Lixia, C. Muscas, and P. Pegoraro, "Impact of the model on the accuracy of synchrophasor measurement," IEEE Transactions on Instrumentation and Measurement, vol. 61, no. 8, pp. 2179-2188, Aug 2012.

[9] D. Belega and D. Petri, "Accuracy analysis of the multicycle synchrophasor estimator provided by the interpolated DFT algorithm," IEEE Transactions on Instrumentation and Measurement, vol. 62, no. 5, pp. 942-953, May 2013.

[10] G. Barchi, D. Macii, D. Belega, and D. Petri, "Performance of synchrophasor estimators in transient conditions: a comparative analysis," IEEE Transactions on Instrumentation and Measurement, pp. 2410-2418, Sept 2013.

[11] T. Routtenberg and L. Tong, "Joint Frequency and Phasor Estimation Under the KCL Constraint," IEEE Signal Processing Letters, vol. 20, no. 6, pp. 575-578, June 2013.
[12] M. Karimi-Ghartemani and M. Iravani, "A nonlinear adaptive filter for online signal analysis in power systems: applications," IEEE Transactions on Power Delivery, vol. 17, no. 2, pp. 617-622, Apr 2002.

[13] R. Naidoo and P. Pillay, "A new method of voltage sag and swell detection," IEEE Transactions on Power Delivery, vol. 22, no. 2, pp. 1056-1063, Apr 2007.

[14] V. Choqueuse, A. Belouchrani, E. El Bouchikhi, and M. Benbouzid, "Estimation of Amplitude, Phase and Unbalance Parameters in Three-phase Systems: Analytical Solutions, Efficient Implementation and Performance Analysis," IEEE Transactions on Signal Processing, vol. 62, no. 16, pp. 4064-4076, Aug 2014.

[15] T. Routtenberg and L. Tong, "Joint frequency and phasor estimation in unbalanced three-phase power systems," in 2014 IEEE International Conference on Acoustics, Speech and Signal Processing (ICASSP), May 2014, pp. 2982-2986.

[16] "IEEE Standard Definitions for the Measurement of Electric Power Quantities Under Sinusoidal, Nonsinusoidal, Balanced, or Unbalanced Conditions," IEEE Std 1459-2010 (Revision of IEEE Std 1459-2000), pp. 1-40, 2010.

[17] S. M. Kay, Fundamentals of Statistical signal processing, Volume 2: Detection theory. Prentice Hall, 1998.

[18] M. E. Tipping and C. M. Bishop, "Probabilistic principal component analysis," Journal of the Royal Statistical Society: Series B (Statistical Methodology), vol. 61, no. 3, pp. 611-622, 1999.

[19] M. H. J. Bollen, "Fast assessment methods for voltage sags in distribution systems," IEEE Transactions on Industry Applications, vol. 32, no. 6, pp. 1414-1423, Nov 1996.

[20] A. Jeffrey and D. Zwillinger, Table of integrals, series, and products. Academic Press, 2007.

[21] S. Kay, Fundamentals of statistical signal processing: Estimation theory. Prentice-Hall, 1993.

[22] A. Renaux, P. Forster, E. Boyer, and P. Larzabal, "Unconditional maximum likelihood performance at finite number of samples and high signal-to-noise ratio," IEEE Transactions on Signal Processing, vol. 55, no. 5, pp. 2358-2364, May 2007. 